\title{
Right-nostril advantage for discrimination of odors
}

\author{
ROBERT J. ZATORRE and MARILYN JONES-GOTMAN \\ Montreal Neurological Institute and Hospital, McGill University, Montreal, Quebec, Canada
}

\begin{abstract}
Olfactory discrimination was tested with eight pairs of odors presented to each nostril of each subject. Ninety-nine subjects were tested; there were equal numbers of left- and right-handers, as well as both sexes. Detection thresholds for phenylethyl alcohol were measured separately in each nostril using a forced-choice staircase procedure. In addition, a verbal dichotic listening test known to be sensitive to language lateralization was administered. Results indicated that discrimination performance was significantly better when the stimuli were presented to the right nostril than when they were presented to the left, but no differences between the nostrils in detection thresholds were found. The right-nostril advantage did not vary as a function of sex or handedness, and did not bear any relation to language lateralization as measured via dichotic listening. The asymmetry for olfactory discrimination replicates an earlier study and is interpreted in terms of a possible specialization of function within the right cerebral hemisphere.
\end{abstract}

Lateral asymmetries in perception of complex stimuli have long been known to exist in the auditory, visual, and somesthetic modalities (see Bryden, 1982, for a comprehensive review). In most cases, these asymmetries have been interpreted as a reflection of a specialization of function in the central nervous system. Thus, for example, in the case of verbal stimuli, a right-ear advantage and a right-visual-field advantage are commonly observed (e.g., Kimura, 1961; McKeever \& Huling, 1971). These effects have been related to left-hemispheric dominance for language function, because the pathways involved are crossed. Conversely, for stimuli that require processing mechanisms presumed to involve right-hemisphere specialization, such as musical sounds, faces, or visuospatial material, the asymmetries tend to favor the left ear or the left visual field (e.g., Kimura, 1964; Rizzolatti, Umilta, \& Berlucchi, 1971; Zatorre, 1979). Although such asymmetries are, at best, an indirect index of the differences between left- and right-hemispheric function, subject to alteration by numerous extraneous variables, they have nevertheless attained importance in neuropsychological studies of normal persons because they are noninvasive, and because they afford insight into one aspect of cerebral function.

In contrast to the extensive work carried out with visual and auditory inputs, very few investigations have examined lateral asymmetries in the olfactory modality, leaving open the question of whether or not hemispheric

This research was supported by Grants MA-10314 and MA-9598 from the Medical Research Council of Canada. We thank Amrita Paul for her assistance in testing the subjects, and Dirk Steenge for help in data tabulation. These results were presented in part at the annual meeting of the Society for Neurosciences, Phoenix, Arizona, November 1989. Correspondence may be addressed to either author, Montreal Neurological Institute, 3801 University St., Montreal, Quebec H3A 2B4, Canada. specialization for olfaction exists. An early study by Toulouse and Vaschide (1900) reported lower detection thresholds for camphor in the left nostril than in the right nostril, but they found lower thresholds in the right nostril for ammonia. More recent data have been contradictory: Koelega (1979) did not find any significant detectionthreshold asymmetries for amyl acetate in a group of 40 right-handed subjects; however, Youngentob, Kurtz, Leopold, Mozell, and Hornung (1981) did find a relation between n-butanol thresholds and handedness in a group of 19 subjects, such that the right nostril was more sensitive in right-handers and the left nostril was more sensitive in left-handers. Pendse (1987) also reported a rightnostril advantage for an intensity scaling task, but only among right-handed women.

In a recent study designed to investigate odordiscrimination ability following focal brain lesions (Zatorre \& Jones-Gotman, in press), we made the unexpected observation that an advantage favoring the right nostril existed in a group of normal right-handed control subjects, despite no significant asymmetries in detection thresholds for n-butanol. We also found that this rightnostril advantage was affected by unilateral cortical excisions: left-temporal-lobe lesions resulted in an exaggerated right-nostril effect, whereas right-temporal-lobe lesions led to a diminished effect. Moreover, bilateral deficits were observed when the cortical damage included the right frontal lobe. Given that the olfactory pathways project ipsilaterally, and given that the two nostrils were equally sensitive on detection thresholds, we interpreted these findings as reflecting a predominance of righthemisphere function specific to discrimination of odors. This conclusion would be consistent with both the lesion effects and the asymmetry observed in normal subjects.

In the present study, it was our aim to address some of the many questions that remain about olfactory asym- 
Table 1

Characteristics of Subject Groups

\begin{tabular}{lccccc}
\hline Group & $N$ & $\begin{array}{c}\text { Mean } \\
\text { Age }\end{array}$ & $\begin{array}{c}\text { Handedness } \\
\text { Score }\end{array}$ & $\begin{array}{c}\text { Number of } \\
\text { Smokers }\end{array}$ & $\begin{array}{c}\text { Familial } \\
\text { Sinistrality }\end{array}$ \\
\hline Female L & 25 & 25.5 & 71.0 & 3 & 8 \\
Female R & 25 & 23.4 & 18.8 & 3 & 3 \\
Male L & 24 & 23.3 & 73.0 & 5 & 7 \\
Male R & 25 & 23.0 & 21.6 & 6 & 13 \\
\hline
\end{tabular}

metries. First, we wanted to see if the nostril difference observed in the previous study with normal subjects would be replicable in a larger sample. Second, we wished to examine the hypothesis that such asymmetries might be related to sex or handedness, two variables that are known to be closely related to hemispheric asymmetries of function. Third, we wanted to assess any possible relationship between olfactory asymmetry and hemispheric specialization for language; therefore, subjects were tested with a verbal dichotic listening test (Wexler \& Halwes, 1983) that is known to be a reliable index of language lateralization (Zatorre, 1989). Finally, to dissociate asymmetries in odor discrimination from any effects related to detection, we measured detection thresholds for phenylethyl alcohol using a forced-choice staircase procedure.

\section{METHOD}

\section{Subjects}

Ninety-nine subjects participated in the study. They were recruited mainly from undergraduate classes at McGill University or from among employees of the Montreal Neurological Hospital, and some were obtained through advertising posters. All were paid for their participation. The subjects were excluded if they had a history of disease or accident that would affect either the central nervous system or the nose. All subjects were free of nasal congestion due to head colds or allergies at the time of testing. No subject was tested within less than $1 \mathrm{~h}$ of eating or drinking. Although smokers were not excluded, few subjects smoked, and they were represented about equally among the experimental groups.

The subjects were divided into four groups, according to sex and handedness. Handedness was assessed using a questionnaire adapted from Crovitz and Zener (1962), which yields a score that can range from 18 (strongly right-handed) to 90 (strongly left-handed); each subject's family history of sinistrality was also recorded. Table 1 shows the mean age and handedness score, together with the incidence of smokers and of subjects with at least one left-handed family member, for each group.

\section{Stimuli and Procedure}

Detection thresholds. Immediately prior to discrimination testing, all subjects were tested for odor-detection thresholds in each nostril using solutions of phenylethyl alcohol diluted in distilled water. This odorant was chosen because of evidence that it is a nearly pure olfactory stimulant, as opposed to a trigeminal stimulant (Doty et al., 1978). The series started at a concentration of $10^{-7.5} \mathrm{M}$ and increased by successive factors of $0.5 \mathrm{log}$ units to a maximum of $10^{-2} \mathrm{M}$. The order of nostril tested first was counterbalanced across subjects. The method followed was similar to that described by Doty, Shaman, and Dann (1984), and involved a staircase procedure to arrive at an estimate of the subject's threshold. Odorants were presented in bottles $(5.8 \mathrm{~cm}$ high, $1.7 \mathrm{~cm}$ in diameter at the opening), filled with approximately $7 \mathrm{ml}$ of liquid. On each trial, the subject sniffed two bottles in turn, one containing a concentration of the odorant and the other containing distilled water, and then indicated which one smelled stronger. The test started with the lowest concentration, which was increased by a factor of 10 (a full $\log$ unit) after each incorrect response until the subject succeeded on four consecutive trials. This value was termed the initial threshold, because it corresponds to the threshold value obtained on a simple ascending series, as used by several authors (e.g., Cain, Gent, Catalanotto, \& Goodspeed, 1983; Eskenazi, Cain, Novelly, \& Mattson, 1986), as well as in one of our own previous studies (JonesGotman \& Zatorre, 1988b).

After four consecutive correct responses, the staircase was reversed, and, from then on, each subsequent concentration differed by a factor of half a log unit instead of one. Thus, upon reaching the initial threshold, the next lower concentration was presented for two trials; if the subject answered correctly on both of these trials, the next lower concentration was again given, but, if an error was made on either trial, the next higher concentration was presented. This procedure continued until seven such reversals were obtained; the final threshold measure was taken to be the geometric mean of the last four staircase reversal points.

Olfactory discrimination testing. Eight pairs of odorants were used, selected from among stimulus pairs that had been rated previously for similarity by normal subjects (Mair, Capra, McEntee, \& Engen, 1980). The extremes were selected for this experiment so that the four pairs that had been rated most highly similar and the four most dissimilar were used. These are listed in Table 2.

These odorants were presented via a modified "sniff strip" (Engen, 1965), a small glass rod with a wad of odor-saturated cotton wrapped around the end. For presentation, the odors were placed under the subject's nostril, providing a more constant vapor than open bottles. The subjects were allowed to sniff according to their preferred strategy, but only one sniff per item presentation was permitted. During the test, the odorants were kept inside a fume adsorber, which filters air through activated charcoal, thereby preventing diffusion of odors into the testing room except during presentation.

The procedure was identical to that used by Zatorre and JonesGotman (in press). The subjects received 32 monorhinal discrimination trials in a same-different paradigm. The 32 trials comprised presentation of each of the eight odor pairs once to each nostril (16 different trials) and presentation of each odor of the pair with itself (16 same trials). The order of presentation of items within each pair was counterbalanced across nostrils; for example, if pair A-B was presented to the left nostril, pair B-A would be presented to the right nostril on a later trial. We tested one nostril on each trial by requiring the subject to hold the other nostril closed with his/her

Table 2

Odorant Pairs Used in Discrimination Task

\begin{tabular}{ll}
\hline \multicolumn{1}{c}{ Similar Pairs } & \multicolumn{1}{c}{ Dissimilar Pairs } \\
\hline Gamma-dodecalactone-Aldehyde C-14 & Anethol-Benzyl butyrate \\
Citronellyl acetate-Citronellyl butyrate & Phenylethyl alcohol-Heptanol \\
Cinnamyl propionate-Cinnamyl butyrate & Oil of cloves-Allyl sulfide \\
Lemon extract-Citral rectified & Butyric acid-Eugenol \\
\hline
\end{tabular}


finger and to inhale only through the open nostril. The order of nostrils tested was randomly assigned across trials. All subjects received the same random order of trials. The two odorants in each trial were presented in succession with a few seconds intervening between the items in a pair; a minimum of $20 \mathrm{sec}$ was allowed between trials.

Dichotic listening. The fused rhymed words test has been described in detail elsewhere (Wexler \& Halwes, 1983). It consists of 15 pairs of words that differ only on the first consonant (e.g., coat/goat, pig/dig). Under normal circumstances, listeners experience a fusion of the two words and typically report hearing only one word at the midline. Stimuli were recorded on cassette tape and played back on a Sony Walkman tape player through matched earphones that had been calibrated to a continuous vowel sound at $75 \mathrm{~dB}(\mathrm{~A})$ using a GenRad $1565-\mathrm{B}$ sound-pressure meter and a type 9A 3-cc acoustic coupler.

The subjects were instructed to choose the one word they had heard out of four possibilities by circling it on a printed page in front of them. Each pair was presented twice per set of trials (once in each of the two possible channel assignments); four randomizations of these 30 trials were presented, for a total of 120 trials (eight repetitions of each pair). The effect of any channel asymmetries was minimized by switching the earphones between ears, once after the first 30 trials and again after 90 trials. Scoring was carried out by eliminating responses that were "stimulus dominated" (i.e., when the subject responds with the same word, regardless of ear of presentation; for details, see Zatorre, 1989). The total number of non-stimulus-dominated responses was then calculated for each ear across all 15 stimulus pairs. These items, which can be considered true asymmetric responses, were used in all subsequent analyses.

\section{RESULTS}

\section{Detection Thresholds}

The average initial and final detection thresholds for each group of subjects are shown in Table 3 . These values were entered into separate ANOVAs, with nostril as a within-subject factor and handedness and sex as betweensubject factors. None of these factors resulted in a significant $F$ ratio, nor were there any significant interactions ( $p>.20$, in all cases). A separate ANOVA was run, with nostril (left or right) and order of testing (left first or right first) as factors. Neither factor resulted in a significant effect. These results indicate, therefore, that the detection thresholds for phenylethyl alcohol did not change as a function of the subject's sex, handedness, nostril tested, or order of testing.

\section{Olfactory Discrimination}

A discrimination score was calculated by subtracting errors (different responses when the items were the same)

Table 3

Mean Oifactory Detection Thresholds: Initial and Final Values of $\mathrm{Log}$ Molar Concentration of Phenylethyl Alcohol

\begin{tabular}{lccccc}
\hline & \multicolumn{2}{c}{ Left Nostril } & & \multicolumn{2}{c}{ Right Nostril } \\
\cline { 2 - 3 } \cline { 5 - 6 } Group & Initial & Final & & Initial & Final \\
\hline Female L & -3.78 & -4.65 & & -3.50 & -4.54 \\
Female R & -3.42 & -4.88 & & -3.80 & -4.91 \\
Male L & -3.38 & -4.58 & & -3.73 & -4.77 \\
Male R & -3.80 & -4.71 & & -3.74 & -4.75 \\
\hline
\end{tabular}

from correct answers (different responses when the items were different), yielding a value with a maximum of 8 . This score, ${ }^{1}$ calculated separately for each nostril, was entered into an ANOVA, with nostril as a within-subject factor and sex, handedness, and familial handedness as between-subject factors. No group differences in overall discriminative ability were observed ( $F<1$, in all cases). As predicted, there was a significant main effect for the nostril factor $[F(1,91)=6.88, p=.01]$, reflecting the better performance obtained on the right nostril (average score of 4.48) as compared with that obtained on the left (average score of 3.89). This result is depicted in Figure 1, which plots the distribution of the difference between the score in the right and left nostrils for each group of subjects.

Although the nostril difference did not interact with either handedness or sex $(p>.10$, in all cases), there was a significant three-way interaction between nostril of presentation, sex, and familial handedness $[F(1,91)=$ $4.58, p<.05]$. Inspection of the means suggests that the male subjects with no history of familial left-handedness yielded, as a group, only a very small difference favoring the right nostril, as compared with the other subgroups. The significance of this effect is difficult to establish, however, as the numbers of observations in each cell were too disparate for post hoc comparisons to be meaningful.

A supplementary analysis was also carried out to determine if there was any difference in the strength of the asymmetry between the similar pairs of odorants and the dissimilar pairs of odorants. For this purpose, the data were rescored separately for similar and dissimilar items, and the discrimination score (with a maximum value of 4) was entered into an ANOVA, with sex and handedness as between-subject factors and nostril and similarity as within-subject factors. The results showed a very large main effect of similarity $[F(1,95)=423.59, p<.0001]$, confirming that the dissimilar items were easier to discriminate. There was also a main effect of nostril $[F(1,95)$ $=5.61, p<.02]$. In addition, there was a significant interaction of nostril $\times$ similarity $[F(1,95)=5.48, p=$ $.02]$. Inspection of the means indicated that the rightnostril advantage was primarily present for the similar items (scores of 0.82 and 1.31 for the left and right, respectively), as compared with the dissimilar items (scores of 3.10 and 3.13 for left and right, respectively).

Finally, to determine if any relationship existed between asymmetries in detection thresholds and asymmetries in discrimination, Pearson product-moment correlations were calculated between the respective difference scores. However, none of these correlations resulted in a statistically significant effect.

\section{Dichotic Listening}

The average scores obtained on each ear in the fused words test, calculated by eliminating all stimulusdominated items (as described in Zatorre, 1989), are shown in Table 4. These scores were entered into an 


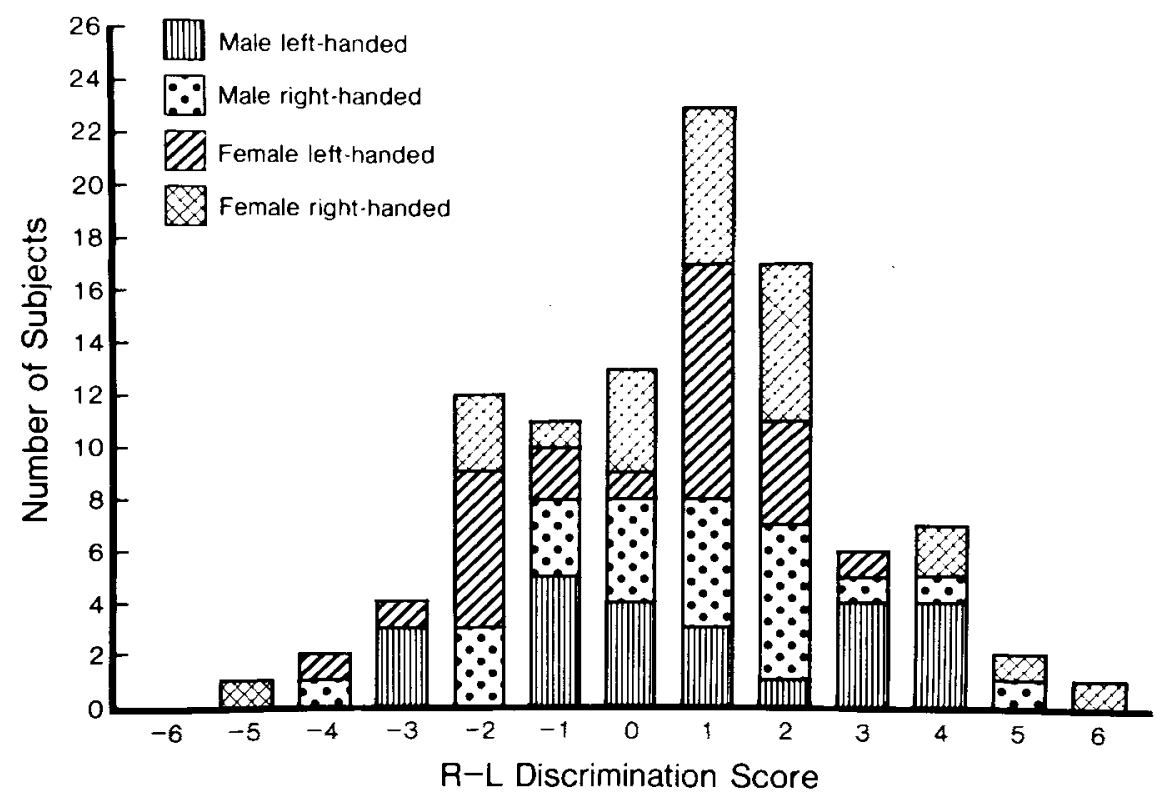

Figure 1. Distribution of asymmetry in olfactory discrimination. The number of subjects obtaining a given difference between the discrimination score in the right and left nostrils is plotted. Positive values along the abscissa indicate right-nostril advantage; negative values indicate left-nostril advantage.

ANOVA, with two between-subject factors, sex and handedness, and one within-subject factor, ear. There was an overall right-ear advantage, as expected $[F(1,95)=37.92$, $p<.0001]$. In addition, this factor interacted with handedness $[F(1,95)=6.76, p=.01]$, such that the lefthanders as a group did not show a significant advantage for the right ear, whereas the right-handers did. There was no interaction with sex $(p>.10)$.

\section{Relation Between Olfactory and Auditory Asymmetries}

To uncover any relation between the right-nostril advantage seen on the olfactory discrimination test and the right-ear advantage seen on the dichotic listening test, a Pearson product-moment correlation coefficient was calculated for the difference scores in each test. The obtained value $(r=-.02)$ was near zero and not significant, suggesting that there is no relation between the two measures.

Table 4

Dichotic Fused Words Test: Number of Words Perceived from Each Ear

\begin{tabular}{lrrrr} 
& \multicolumn{2}{c}{ Left Ear } & & \multicolumn{2}{c}{ Right Ear } \\
\cline { 2 - 3 } \cline { 5 - 5 } Group & \multicolumn{1}{c}{$M$} & $S D$ & & $M$ \\
\hline Female L & 11.4 & 8.4 & 12.0 & \multicolumn{1}{c}{$S D$} \\
Female R & 5.4 & 5.3 & 18.5 & 10.9 \\
Male L & 9.9 & 10.9 & 19.6 & 12.8 \\
Male R & 6.1 & 5.3 & 18.6 & 11.3 \\
\hline
\end{tabular}

Note-Values were calculated by eliminating all items that were stimulusdominated (see Zatorre, 1989).
Another way to examine this question is to select only those subjects whose dichotic listening scores are sufficiently extreme that one may infer, with relative certainty, their language lateralization, irrespective of handedness. On the basis of previous validation data (Zatorre, 1989), a criterion was set according to the difference in number of words reported between the right and left ears. Only the subjects obtaining a difference of 20 words or greater favoring the right ear or 5 words or greater favoring the left ear were retained. This yielded 21 subjects in the first group (those whose language is likely to be lateralized to the left), and 10 in the second (those presumed to have right-sided, or possibly bilateral, language representation). The difference between nostrils in the discrimination test was compared in these subgroups of patients, and was found to be not significantly different $[t(29)=0.36]$. The difference favored the right nostril in both subgroups: for the group with large right-ear advantages, the mean nostril difference was 0.47 ; for the group with left-ear advantages, the mean nostril difference was 0.80 .

\section{DISCUSSION}

The results of this study clearly demonstrate a perceptual asymmetry in olfactory discrimination that was reliably present in the population, although it was not observed for every individual. Moreover, the advantage of the right nostril was observed in the absence of any threshold differences, and it did not seem to be related to sex, handedness, or side of presumed language repre- 
sentation. These data replicate and extend our earlier finding of a right-nostril advantage for the same task (Zatorre \& Jones-Gotman, in press). Having established the existence of such an asymmetry, its nature and origin remain to be explained.

One might argue that some difference at the periphery of the olfactory system could account for our results. However, there is little evidence in favor of such a hypothesis. First, there are no studies of peripheral anatomical structures, to our knowledge, that have revealed any striking structural asymmetries favoring one side over the other. Furthermore, the fact that we did not find any significant difference in detection thresholds between the two nostrils, for either the initial or the final threshold estimate, would also argue that the discrimination results are not due to a peripheral component, but rather are related to central processes. It should be pointed out that we undertook a staircase procedure to measure threshold detection because of evidence that a simple ascending method of limits results in marked subject-to-subject and study-to-study variability (Doty, Gregor, \& Settle, 1986). We also wished to compare the results with our previous finding (Jones-Gotman \& Zatorre, 1988b) that the second nostril tested tended to have a lower threshold than did the first, presumably due to some sort of practice effect. Our present results confirm that the final threshold estimate is much lower than is the initial value (Table 3 ), but we did not observe any order effects, reflecting the greater stability of the measure obtained via this technique.

Youngentob et al. (1981) did report a difference in detection thresholds favoring the right nostril in righthanders and the left in left-handers. However, they also found that subjects whose nasal patency was slightly less on one side had lower thresholds on that side, but it was not clear if this accounted for their reported interaction with handedness. Nasal patency would also be affected by cyclical changes in nasal airflow (Principato \& Ozenberger, 1970), but recent evidence indicates that such changes are not very regular and do not favor one nostril over the other in any systematic fashion (Gilbert \& Rosenwasser, 1987). Whether the results of Youngentob et al. bear any relation to the phenomenon uncovered in the present study remains a question for future research.

Classical anatomical studies have established that the olfactory nerve projects ipsilaterally to the olfactory bulb, which in turn projects to ipsilateral olfactory cortical areas (for a review, see Eslinger, Damasio, \& Van Hoesen, 1982). If the present results reflect an asymmetry in central olfactory processing, it is reasonable to assume that certain regions in the right cerebral hemisphere may show at least a partial functional superiority to homologous areas in the left hemisphere. It should be pointed out, however, that the discrimination task may also have involved some degree of stimulation of the trigeminal nerve, whose projection is crossed. Although the task required a qualitative discrimination, and therefore presumably depended primarily on input from the first cranial nerve, a trigeminal contribution cannot be ruled out, especially for cer- tain pairs of stimuli, such as those that differed significantly in perceived strength. Any information conveyed to the contralateral hemisphere via the trigeminal nerve would serve to obscure or dilute the asymmetry due to olfactory nerve input. In this respect, it is notable that the similar (and therefore more difficult to discriminate) items were the ones that elicited the clearer right-nostril advantage. We may speculate that, among the dissimilar items, there were some pairs that could be distinguished on the basis of strength alone, and that this may have led to a diminished asymmetry since both ipsilateral and contralateral inputs could be used to respond correctly. On the other hand, the asymmetry may have been reduced because performance was near ceiling with the dissimilar items. Future studies will have to clarify the contribution of olfactory versus trigeminal input to asymmetries in odor discrimination.

The dichotic listening results were entirely in keeping with previous studies: a clear right-ear advantage was found among the right-handers, whereas the left-handers as a group did not evidence a clear ear difference. It is notable that we found no evidence of a relationship between asymmetries in language function, as measured via dichotic listening, and the olfactory asymmetry. It might be argued that the lack of correlation is not surprising since laterality measures across modalities rarely correlate very well, even when both involve language function (Hines \& Satz, 1974; Zurif \& Bryden, 1969). However, the dichotic fused words test has been shown to be highly reliable (Wexler \& Halwes, 1983) and has furthermore been shown to be closely related to hemispheric language dominance as measured independently via intracarotid sodium Amytal testing (Zatorre, 1989). Moreover, even if we ignore degree of asymmetry and choose only those subjects whose dichotic listening scores are sufficiently extreme to infer, with relative certainty, their language laterality, we still find no relation to olfactory asymmetry. In fact, both right- and left-ear-dominant subjects showed equal right-nostril advantages. These facts, together with the lack of effect of handedness on the olfactory asymmetry, lead us to conclude that the olfactory discrimination results reflect specialization of hemispheric function that is independent of language.

The suggestion that there may be some degree of specialization within the right hemisphere for certain types of olfactory processing receives support from a number of independent sources, notably from neuropsychological studies of olfactory function following brain lesions. Several authors have reported findings suggesting that right temporal-lobe or orbitofrontal lesions lead to more marked deficits than do similar lesions in the left hemisphere in odor matching or recognition tasks (Abraham \& Mathai, 1983; Jones-Gotman \& Zatorre, 1988a; Rausch, Serafetinides, \& Crandall, 1977). On the other hand, neither Eskenazi and colleagues (Eskenazi, Cain, Novelly, \& Friend, 1983; Eskenazi et al., 1986) nor Jones-Gotman and Zatorre (1988b) reported significant differences related to side of excision in several other ol- 
factory tasks. If a functional hemispheric asymmetry exists, it would appear to be a relative one: both hemispheres are likely involved, but perhaps to different degrees. This conclusion would be consistent with most of the evidence showing that lesions to olfactory regions in either hemisphere usually lead to deficits. It would also be in agreement with reports of olfactory matching in patients with section of the corpus callosum (Gordon \& Sperry, 1969), who are indeed able to carry out such a task with input to either nostril, so long as the matches presented to the right nostril can be done in a nonverbal manner.

There is also a recent report with normal subjects (Richardson \& Zucco, 1989) of faster responses to odor-visual matches when the visual stimuli were presented to the left visual field than when they were presented to the right visual field, implying a righthemisphere superiority for this type of cross-modal judgment. It remains to be established whether the right-nostril advantage uncovered in the present study is limited to tasks that involve qualitative discrimination of odors, or whether it might also be found with other types of tasks, reflecting some more general aspect of olfactory function.

\section{REFERENCES}

Abraham, A., Mathai, K.V. (1983). The effect of right temporal lobe lesions on matching of smells. Neuropsychologia, 21, 277-281.

Bryden, M. P. (1982). Laterality: Functional asymmetry in the intact brain. New York: Academic Press.

Cain, W. S., Gent, J., Catalonotto, F. A., \& Goodspeed, R. B. (1983). Clinical evaluation of olfaction. American Joumal of Otolaryngology, 4, 252-256.

Crovitz, H. F., \& Zener, K. (1962). A group test for assessing handand eye-dominance. American Journal of Psychology, 75, 271-276.

Doty, R. L., Brugger, W. E., Jurs, P. C., Orndorff, M. A., SNYDER, P. J., \& LoWRY, L. D. (1978). Intranasal trigeminal stimulation from odorous volatiles: Psychometric responses from anosmic and normal humans. Physiology \& Behavior, 20, 175-185.

Doty, R. L., Gregor, T. P., \& SETtLe, R. G. (1986). Influence of intertrial interval and sniff-bottle volume on phenyl ethyl alcohol odor detection thresholds. Chemical Senses, 11, 259-264.

Doty, R. L., Shaman, P., DanN, M. (1984). Development of the University of Pennsylvania Smell Identification Test: A standardized microencapsulated test of olfactory function. Physiology \& Behavior, 32, 489-502.

ENGEN, T. (1965). Psychophysical analysis of the intensity of homologous alcohols. Journal of Experimental Psychology, 70, 611-616.

Eskenazi, B., Cain, W. S., Novelly, R. A., \& Friend, K. B. (1983). Olfactory functioning in temporal lobectomy patients. Neuropsychologia, 21, 365-374.

Eskenazi, B., Cain, W. S., Novelly, R. A., \& Mattson, R. (1986). Odor perception in temporal lobe epilepsy patients with and without temporal lobectomy. Neuropsychologia, 24, 553-562.

Eslinger, P. J., Damasio, A. R., \& Van Hoesen, G. W. (1982). Olfactory dysfunction in man: Anatomical and behavioral aspects. Brain \& Cognition, 1, 259-285.

Gilbert, A. N. , Rosenwasser, A. M. (1987). Biological thythmicity of nasal airway patency: A re-examination of the 'nasal cycle." Acta Otolaryngologica, 104, 180-186.

GORDON, H. W., \& SPERRY, R. W. (1969). Lateralization of olfactory perception in the surgically separated hemispheres of man. Neuropsychologia, 7, 111-120.

Hines, D., \& SATZ, P. (1974). Cross-modal asymmetries in perception related to asymmetry in cerebral function. Neuropsychologia, 12, 239-247.

Jones-Gotman, M., \& Zatorre, R. J. (1988a). Contribution of right temporal lobe to odor memory. Epilepsia, 29, 661 .

Jones-Gotman, M., \& ZATORRE, R. J. (1988b). Olfactory identification deficits in patients with focal cerebral excision. Neuropsychologia, 26, 387-400.

Kimura, D. (1961). Cerebral dominance and the perception of verbal stimuli. Canadian Journal of Psychology, 15, 166-171.

Kimura, D. (1964). Left-right differences in the perception of melodies. Quarterly Journal of Experimental Psychology, 16, 355-358.

Koelega, H. S. (1979). Olfaction and sensory asymmetry. Chemical Senses \& Flavor, 4, 89-95.

Mair, R., Capra, C., McEntee, W. J., \& Engen, T. (1980). Odor discrimination and memory in Korsakoff's psychosis. Journal of Experimental Psychology: Human Perception \& Performance, 6 , 445-458.

MCKeEver, W., \& Huling, M. D. (1971). Bilateral tachistoscopic word recognition as a function of hemisphere stimulated and interhemispheric transfer time. Neuropsychologia, 9, 15-20.

Pendse, S. G. (1987). Hemispheric asymmetry in olfaction on a category judgment task. Perceptual \& Motor Skills, 64, 495-498.

Principato, J. J., \& Ozenberger, J. M. (1970). Cyclic changes in nasal resistance. Archives of Otolaryngology, 91, 71-77.

Rausch, R., Serafetinides, E. A., \& Crandall, P. H. (1977). Olfactory memory in patients with anterior temporal lobectomy. Cortex, 13, 445-452.

Richardson, J. T. E., \& Zucco, G. M. (1989). Cognition and olfaction: A review. Psychological Bulletin, 105, 352-360.

Rizzolatti, G., Umilta, C., \& Berlucchi, G. (1971). Opposite superiorities of the right and left cerebral hemispheres in discriminative reaction time to physiognomical and alphabetical material. Brain, 94, 431-442.

TOUlOuSE, E., \& VASChIDE, N. (1900). L'asymetrie sensorielle olfactive. Revue Philosophique, 49, 176-187.

WEXLER, B. E., HALWES, T. (1983). Increasing the power of dichotic methods: The fused thymed words test. Neuropsychologia, 21, 59-66.

Youngentor, S. L., KuRTZ, D. B., Leopold, D. A., Mozell, M. M., \& HoRnUNG, D. E. (1981). Olfactory sensitivity: Is there laterality? Chemical Senses \& Flavor, 6, 11-21.

ZATORRE, R. J. (1979). Recognition of dichotic melodies by musicians and nonmusicians. Neuropsychologia, 17, 607-617.

ZATORRE, R. J. (1989). Perceptual asymmetry on the dichotic fused words test and cerebral speech lateralization determined by the carotid Amytal test. Neuropsychologia, 27, 1207-1219.

ZatoRRE, R. J., \& JoNeS-GotMaN, M. (in press). Human olfactory discrimination after unilateral frontal or temporal lobectomy. Brain.

ZURIF, E. B., Bryden, M. P. (1969). Familial handedness and leftright differences in auditory and visual perception. Neuropsychologia, 7, 179-187.

\section{NOTE}

1. This score was used because of its simplicity and for comparison with other data, even though it is not an unbiased estimate of discrimination ability. Accordingly, the results were also evaluated with $A^{\prime}$, a distribution-free index of discriminability. However, the results using $A^{\prime}$ were identical in all respects to the results using the simpler discrimination score, and so will not be reported here.

(Manuscript received January 8, 1990; accepted for publication January 17,1990 .) 